\title{
Gas rarefaction effects during high power pulsed magnetron sputtering of groups IVb and VIb transition metals in Ar
}

Grzegorz Greczynski, Igor Zhirkov, Ivan Petrov, J oseph E Greene and J ohanna Rosén

The self-archived postprint version of this journal article is available at Linköping University Institutional Repository (DiVA):

http:/ / urn.kb.se/ resolve?urn=urn:nbn:se:liu:diva- 143365

N.B.: When citing this work, cite the original publication.

Greczynski, G., Zhirkov, I., Petrov, I., Greene, J. E, Rosén, J., (2017), Gas rarefaction effects during high power pulsed magnetron sputtering of groups IVb and VIb transition metals in Ar, Journal of Vacuum Science \& Technology. A. Vacuum, Surfaces, and Films, 35(6), .

https:// doi.org/ 10.1116/ 1.4989674

Original publication available at:

https:// doi.org/ 10.1116/ 1.4989674

Copyright: American Institute of Physics (AIP)

https:// www.aip.org/ 


\title{
Gas rarefaction effects during high power pulsed magnetron sputtering of Groups IVb and VIb transition metals in Ar
}

\author{
G. Greczynski, ${ }^{1}$ I. Zhirkov, ${ }^{1}$ I. Petrov, ${ }^{1,2}$ J.E. Greene, ${ }^{1,2,3}$ and J. Rosen ${ }^{1}$ \\ ${ }^{1}$ Department of Physics (IFM), Linköping University, SE-581 83 Linköping, Sweden \\ ${ }^{2}$ Materials Science Department and Frederick Seitz Materials Research Laboratory, University of \\ Illinois, Urbana, Illinois 61801 \\ ${ }^{3}$ Department of Physics, University of Illinois, Urbana, Illinois 61801, USA
}

\begin{abstract}
We use energy- and time-dependent mass spectrometry to analyze the evolution of metaland gas-ion fluxes incident at the substrate during high-power pulsed magnetron sputtering (HiPIMS) of Groups IVb and VIb transition-metal (TM) targets in Ar. For all TMs, the time-and energy-integrated metal/gas-ion ratio at the substrate plane $N_{M e^{+}} / N_{A r^{+}}$increases with increasing peak target current density $J_{T, p e a k}$ due to rarefaction. In addition, $N_{M e^{+}} / N_{A r^{+}}$exhibits a strong dependence on metal/gas-atom mass ratio $m_{M e} / m_{g}$ and varies from $\sim 1$ for $\mathrm{Ti}\left(m_{T i} / m_{A r}=1.20\right)$ to 100 for $\mathrm{W}\left(m_{W} / m_{A r}=4.60\right)$, with $J_{T \text {,peak }}$ maintained constant at $1 \mathrm{~A} / \mathrm{cm}^{2}$. Time-resolved IEDFs confirm that the degree of rarefaction scales with $m_{M e} / m_{g}$ : for heavier TMs, the original sputteredatom Sigmund-Thompson energy distributions are preserved long after the HiPIMS pulse, which is in distinct contrast to lighter metals for which the energy distributions collapse into a narrow thermalized peak. Hence, precise timing of synchronous substrate-bias pulses, applied in order to reduce film stress while increasing densification, is critical for metal/gas combinations with $m_{M e} / m_{g}$ near unity, while with $m_{M e} / m_{g} \gg 1$, the width of the synchronous bias pulse is essentially controlled by the metal-ion time of flight. The good agreement between results obtained in an industrial system employing $440 \mathrm{~cm}^{2}$ cathodes and a laboratory-scale system with a $20 \mathrm{~cm}^{2}$ target is indicative of the fundamental nature of the phenomena.
\end{abstract}

corresponding author: grzgr@ifm.liu.se; phone: +4613281213 


\section{INTRODUCTION}

High-power pulsed magnetron sputtering (HiPIMS) ${ }^{1,2}$ provides an alternative route to that of magnetically-unbalanced magnetron sputtering ${ }^{3,4}$ for ion-assisted transition-metal (TM) nitride film growth due to the inherent separation of metal- and gas-ion fluxes that stems from gas rarefaction. ${ }^{5}$ The latter effect provides the ability to separate, in both time and energy domains, metal ions and gas ions incident at the substrate, by employing a substrate bias potential that is synchronized with the metal-ion-rich portion of the HiPIMS pulse. ${ }^{6,7}$ Metal-ion-synchronized HiPIMS deposition opens new film-growth pathways and provides additional control of film composition and properties. Metal-ions, as opposed to noble-gas ions, are primarily incorporated at lattice sites which, together with dramatically-reduced concentrations of trapped gas ions, results in lower compressive stresses in as-deposited layers. Moreover, the metal-ion mass, incident flux, and impact energy can be independently controlled to optimize momentum transfer and provide the recoil density and energy necessary to eliminate film porosity at low deposition temperatures.

Metal-ion-synchronized HiPIMS has been used to grow nanostructured N-doped bcc$\mathrm{CrN}_{0.05}$ films possessing atomically-smooth surfaces and combining properties which are characteristic of both metals (bcc-Cr crystal structure, electrical resistivity, and toughness) and ceramics (high hardness). ${ }^{8}$ By operating synchronized-HiPIMS jointly with a dc magnetron in a co-sputtering configuration to grow pseudobinary nitrides, the effect of each metal-ion can be probed separately. ${ }^{9,10}$ We demonstrated fully-dense, hard, and stress-free $\mathrm{Ti}_{0.39} \mathrm{Al}_{0.61} \mathrm{~N},{ }^{11}$ singlephase cubic $\mathrm{Ti}_{1-x} \mathrm{Si}_{x} \mathrm{~N}$ with record-high $\mathrm{SiN}$ concentrations, ${ }^{12}$ unprecedented AlN supersaturation in single-phase $\mathrm{NaCl}$-structure $\mathrm{V}_{1-x} \mathrm{Al}_{x} \mathrm{~N},{ }^{13}$ and a dramatic increase in the hardness of dense TM nitride films deposited with no external heating $\left(T_{s}<12 \%, T_{s} / T_{m}<12 \% ; T_{s}\right.$ and $T_{m}$ are the film growth and melting temperatures, respectively, in K) due to selective heavy-metal-ion 
bombardment during growth of dilute $\mathrm{Ti}_{0.92} \mathrm{Ta}_{0.08} \mathrm{~N}$ alloys. ${ }^{14,15}$ The technique relies on detailed knowledge of the time evolution of metal- and gas-ion fluxes at the substrate in order to set the synchronous bias pulse.

Here, we use energy- and time-dependent mass spectrometry to analyze the evolution of metal- and gas-ion fluxes incident at the substrate during and after HiPIMS pulses while sputtering Groups IVb and VIb TM targets in Ar. We show that the time- and energy-integrated metal/gasion ratio $N_{M e^{+}} / N_{A r^{+}}$incident at the substrate plane increases with increasing peak target current density $J_{T, p e a k}$ as a result of sputter-gas rarefaction. Moreover, the effect scales with increasing metal-ion mass, resulting in $N_{M e^{+}} / N_{A r^{+}}$varying by two orders of magnitude from $\sim 1$ for Ti $\left(m_{T i}\right.$ $=47.87 \mathrm{amu})$ to $\sim 100$ for $\mathrm{W}\left(m_{W}=183.84 \mathrm{amu}\right)$.

\section{EXPERIMENTAL PROCEDURE}

Time-dependent in-situ mass- and energy-spectroscopy analyses of ion fluxes during HiPIMS sputtering of Ti, Zr, Hf, Cr, Mo, and W targets (99.99 \% pure) in Ar are performed using a Hiden Analytical EQP1000 instrument. Experiments are carried out in a CemeCon CC800/9 magnetron sputtering system equipped with rectangular $8.8 \times 50 \mathrm{~cm}^{2}$ targets parallel to, and $18 \mathrm{~cm}$ from, the orifice of the mass spectrometer at the normal substrate position. The system base pressure is 0.2 $\mathrm{mPa}\left(1.5 \times 10^{-6}\right.$ Torr), and the Ar sputtering pressure is maintained constant at $P=0.4 \mathrm{~Pa}$ ( $3 \mathrm{mTorr}$ ). The HiPIMS pulse length is $120 \mu$ s at a frequency of $300 \mathrm{~Hz}$. Ion-energy distribution functions $I_{M e^{n+}}\left(E_{i}\right)$ (IEDFs) are recorded in HiPIMS mode for $\mathrm{Me}^{n+}(n=1,2, \ldots)$ metal and gas ions during 100 consecutive pulses such that the total acquisition time per data point is $1 \mathrm{~ms}$. The ion energy is scanned in $0.5 \mathrm{eV}$ steps from $E_{i}=1$ to $50 \mathrm{eV}$. Additional details regarding the IEDF measurements, including ion time-of-flight corrections, are given in Refs. 16,17. 


\section{RESULTS AND DISCUSSION}

Time-dependent intensities of the primary ion fluxes recorded during Ti-HiPIMS $\left(\mathrm{Ti}^{+}, \mathrm{Ti}^{2+}\right.$, and $\mathrm{Ar}^{+}$) are plotted in Fig. 1 with a $10 \mu$ s resolution for selected values of the peak target current density $J_{T \text {,peak }}$ ranging from 0.03 to $2.0 \mathrm{~A} / \mathrm{cm}^{2}$. Zero on the time axis corresponds to the onset of the cathode voltage pulse, while each data point at time $t$ represents the number of ions collected during the interval from $t-5$ to $t+5 \mu$ s. The time-separation of metal- and gas-ion fluxes strongly depends on the peak target current density. This is also true for all other transition metals investigated. With $J_{T, p e a k}=0.03 \mathrm{~A} / \mathrm{cm}^{2}$, a value characteristic of dc magnetron sputtering (DCMS), the ion flux at the substrate plane is dominated by $\mathrm{Ar}^{+}$. The maximum intensity of the gas-ion flux $F_{A r^{+}}(t)$, obtained at $t=130 \mu \mathrm{s}$, coincides with the peak position of the Ti-ion flux $F_{T i^{+}}(t)$; however, the latter is $\sim 3 \times$ less intense. Both ion fluxes decay to nearly zero at $t=200 \mu$ s.

For $J_{T, p e a k}$ from 0.1 to $0.5 \mathrm{~A} / \mathrm{cm}^{2}$, current densities corresponding to the transition region between DCMS and HiPIMS, the metal-ion flux increases substantially and $F_{T i^{+}}(t)$ peak values now exceed those of $F_{A r^{+}}(t)$. More importantly, the time separation between the metal- and gas-ion fluxes increases. For example, with $J_{T, p e a k}=0.5 \mathrm{~A} / \mathrm{cm}^{2}, F_{T i^{+}}(t)$ completely dominates the ion flux to the substrate from 30 to $100 \mu$ s. Independent of $J_{T, p e a k}$, the peaks in $F_{T i^{+}}(t)$ are due to faster $\mathrm{Ti}^{+}$ions with an average energy $\bar{E}_{\iota}$ of $10.5 \mathrm{eV}$, while the longer-time tails extending as far as $300 \mu$ s are due to low-energy (thermalized) ions (see below) with $\bar{E}_{l}=2.0 \mathrm{eV}$. The peak in $F_{T i^{+}}(t)$ moves toward $t=0$ with increasing $J_{T, p e a k}$, while the $F_{A r^{+}}(t)$ ion distribution, very broad (100 to $350 \mu$ s) with $J_{T, p e a k}=0.1 \mathrm{~A} / \mathrm{cm}^{2}$, narrows to $80-200 \mu$ s, as the peak target current density is increased to $0.5 \mathrm{~A} / \mathrm{cm}^{2}$. These effects are the consequence of the narrowing of the HiPIMS current pulse as the discharge becomes power-supply limited. 
At even higher peak target currents, $1.0-2.0 \mathrm{~A} / \mathrm{cm}^{2}$, characteristic of HiPIMS, $F_{T i^{+}}(t)$ forms a relatively narrow peak which dominates the ion flux to the substrate from $t=30$ to $90 \mu \mathrm{s}$. A new feature in the $F_{\mathrm{Ar}^{+}}(t)$ time evolution, in agreement with previous reports, ${ }^{5,6}$ is the appearance of peaks in the early stage, $0-40 \mu \mathrm{s}$, indicating that the plasma is initially maintained primarily by gas ions. After a rapid decay of the metal-ion flux, $F_{A r^{+}}(t)$ again dominates the ion flux from $t=100$ to $200 \mu$ s as the Ar density increases in front of the sputtering target due to a rapid decrease in $J_{T, p e a k}$

Fig. 2 shows time-dependent intensities of the primary-ion fluxes incident at the substrate

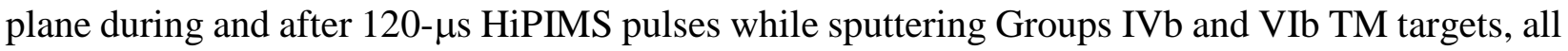
operated at a target current density of $1 \mathrm{~A} / \mathrm{cm}^{2}$. Clearly, for both Groups, the $\mathrm{Ar}^{+}$ion flux decreases with increasing metal-ion mass. In the case of IVb metals, the energy and time-integrated metal/gas-ion ratio $N_{M e^{+}} / N_{A r^{+}}$, defined as $\int F_{M e^{+}}(t) d t / \int F_{A r^{+}}(t) d t$ with the integral extending from $t=0$ to $300 \mu \mathrm{s}$, increases from 1.2 for $\mathrm{Ti}\left(m_{T i}=47.87 \mathrm{amu}\right)$, to 8.3 for $\mathrm{Zr}\left(m_{Z r}=91.22 \mathrm{amu}\right)$, and 30.1 for $\mathrm{Hf}\left(m_{H f}=178.49 \mathrm{amu}\right)$. In a similar manner, $N_{M e^{+}} / N_{A r^{+}}=10$ for $\mathrm{Cr}\left(m_{C r}=52.00\right.$ amu), and increases to 40 and 110 for Mo $\left(m_{M o}=95.94 \mathrm{amu}\right)$ and $\mathrm{W}\left(m_{W}=183.84 \mathrm{amu}\right)$, respectively. There are two possible phenomena which could account for the observed loss of $F_{A r^{+}}(t)$ intensity: (i) gas rarefaction, ${ }^{18,19,20,21}$ and (ii) changes in the plasma electron temperature induced by electron-impact ionization of sputter-ejected metal atoms that have low first-ionization potentials $I P_{M e}^{1}$ resulting in reduced intensity in the high-energy tail in the plasma electron energy distribution which provides, in turn, a decrease in the ionization probability of $\operatorname{Ar}\left(I P_{A r}^{1}=15.75\right) .{ }^{22}$ If the later were the dominant effect, the $\mathrm{N}_{\mathrm{Me}^{+}} / \mathrm{N}_{\mathrm{Ar}^{+}}$ratio should be similar for all Group IVb TMs since they have very similar $I P_{M e}^{1}$ values (see Table 1 ). The strong correlation between $N_{M e^{+}} / N_{A r^{+}}$ and $m_{M e}$ indicates that gas rarefaction dominates. Higher $N_{M e^{+}} / N_{A r^{+}}$values for VIb than IVb 
metals (comparing elements with similar mass) can be ascribed to higher sputter yields $S_{A r \rightarrow M e}$ and $S_{M e \rightarrow M e}$ (see Table 1) which result in higher metal fluxes per unit time, leading to increased momentum transfer to gas atoms and, hence, stronger rarefaction.

Another important observation for metal-ion-synchronized HiPIMS is that the time delays between the maxima in target current and subsequent $F_{M e^{+}}(t)$ peaks increase with increasing metal-ion mass; from $30 \mu$ s for Ti and Cr, to $48 \mu$ s for Zr and Mo, and $63 \mu$ s for Hf and W. Consequently, metal-ion peaks are broader for heavier ions. The width of the $F_{M e^{+}}(t)$ distribution at $10 \%$ intensity, which includes the entire time period over which metal ions are incident at the substrate, varies from 100 $\mu$ s for Ti and Cr, to 140 $\mu$ s for Zr and Mo, and 200 $\mu$ s for Hf and W. Both effects stem from the fact that the time-of-flight from the target increases with increasing $m_{M e}$.

The above results have direct implications for HiPIMS film growth employing synchronous biasing. First, precise tuning of bias pulse length and offset, used to control the energy and momentum of metal ions incident at the growing film surface, is more critical when the masses of sputtered metal species and gas atoms are similar, e.g. Ti/Ar and $\mathrm{Cr} / \mathrm{Ar}$ in these experiments. For such cases, the pulse offset and length necessary to obtain the best time separation between metaland gas-ion fluxes at the substrate plane depend strongly on the HiPIMS peak target current (see Fig. 1). At higher $m_{M e} / m_{g}$ ratios, $N_{M e^{+}} / N_{A r^{+}}$increases rapidly due to rarefaction and the ion flux to the substrate becomes dominated by metal ions. Thus, there is more flexibility in choosing pulse shapes in order to maximize the metal-ion flux while minimizing gas-ion irradiation incident at the substrate. The bias pulse width can be increased with increasing ion mass for a given target/substrate separation (see Fig. 2). 
$N_{\mathrm{Me}^{+}} / N_{\mathrm{Ar}}+$ ratios are plotted as a function of peak target current density $J_{T, p e a k}$ for all Groups IVb and VIb TMs in Fig. 3. The trends outlined above for the case of $J_{T, p e a k}=1 \mathrm{~A} / \mathrm{cm}^{2}$ hold over the entire current range. Within each Group, $N_{\mathrm{Me}^{+}} / N_{\mathrm{Ar}^{+}}$increases with increasing atomic mass and, for a given element, $N_{M e^{+}} / N_{A r^{+}}$increases continuously with $J_{T, p e a k}$.

Fig. 4 presents $\mathrm{Me}^{+}$IEDFs for TMs recorded during consecutive 50- $\mu$ s time intervals at the substrate plane during and after 120- $\mu$ s HiPIMS pulses while sputtering in Ar. Since metal IEDFs depend upon mass transport through the gas phase, analyses of $I_{M e^{+}}\left(E_{i}\right)$ time evolutions reveal essential differences among the metals. For the lighter TM metals, Ti (in particular) and Cr, metalion IEDFs in the early stages $(t<100 \mu \mathrm{s})$, resemble very broad Sigmund-Thompson sputteredspecies energy distributions; ${ }^{23,24}$ that is, $I_{T i^{+}}\left(E_{i}\right) \propto E_{i} /\left(E_{i}+E_{b}\right)^{3}$ (in which $E_{b}$ denotes the surface-atom binding energy). ${ }^{23}$ Thus, the original energy distribution of sputter-ejected atoms is preserved since these species undergo few or no collisions due to gas rarefaction. ${ }^{25}$ During the later phases ( $t>150 \mu \mathrm{s}), I_{T i^{+}}\left(E_{i}\right)$ IEDFs collapse to narrow, low-energy peaks at 2-3 eV (reflecting the potential difference between the bulk plasma potential and the grounded orifice), as a result of thermalization, in which the sputtered species lose energy via collisions with noble-gas atoms, as the target current density and, hence, rarefaction decreases (i.e. local rare-gas pressure increases).

The thermalization distance $\lambda_{\text {th }}$ for sputtered species ejected normal to the target, the distance at which the initial velocity $v_{o}$ is reduced to the thermal velocity $v_{t h}$, is given approximately by the expression $^{26}$

$$
\lambda_{t h}=\lambda \eta=\lambda \ln \left(v_{t h} / v_{o}\right) / \ln (\xi),
$$

in which $\lambda$ is the mean free path, $\eta$ is the number of collisions in the ejection direction required to thermalize a sputter-ejected atom, and $\xi$ depends only on the ratio of the sputter-ejected atom mass to that of the sputtering gas. ${ }^{27}$ Estimated $\eta, \lambda$, and $\lambda_{\text {th }}$ values for metal atoms passing through $\mathrm{Ar}$ 
discharges at $P=3$ mTorr with an average sputter-ejection energy of $10 \mathrm{eV}$ (see Fig. 4) are listed in Table 1. The choice of ion energy is based on time-resolved IEDFs discussed in detail below. Estimates of $\lambda_{\text {th }}$ based on Eq. (1) should be treated as lower limits since gas rarefaction effects, which are strong in HiPIMS due to high instantaneous sputter-ejected fluxes, further extend the thermalization distance since $\lambda$ which is $\propto 1 / P$.

IEDFs in Fig. 4 reveal that rarefaction scales with increasing metal/gas-atom mass ratio $m_{M e} / m_{g}$. For heavier TMs, Zr and Mo, the original $I_{M e^{+}}\left(E_{i}\right)$ with broad Sigmund-Thompson energy distributions are preserved throughout the entire measurement period up to $300 \mu$ s, and beyond. The $I_{\mathrm{Me}^{+}}\left(E_{i}\right)$ IEDFs do not collapse into a narrow thermalized peak, as observed for $\mathrm{Ti}^{+}$. Instead, a gradual loss in intensity occurs and even the ions arriving within the time interval 250$300 \mu$ s have an average energy of 12 ( $\mathrm{Zr}$ ) to $15 \mathrm{eV}$ (Mo). The dramatic decrease in thermalized ion density, despite the fact that $\lambda_{\text {th }}$ is significantly shorter than the target-orifice distance (see Table 1), indicates severe rarefaction and is consistent with the fact that the energy and time-integrated metal/gas-ion ratio $\mathrm{N}_{\mathrm{Me}^{+}} / \mathrm{N}_{\mathrm{Ar}^{+}}$increases with increasing metal-ion mass, as discussed above. Severe rarefaction is due to the fact that the metal/gas collision cross-section increases with increasing sputtered atom mass, resulting in shorter mean free paths (see Table 1), increased momentum transfer, and hence more effective gas heating. The process is governed by the $m_{M e} / m_{g}$ ratio. Previous results indicate that the thermalized $\mathrm{Ti}^{+}$ion peak disappears if $\operatorname{Ar}\left(m_{A r}=\right.$ $39.95 \mathrm{amu})$ is replaced with $\mathrm{Ne}\left(m_{N e}=20.18 \mathrm{amu}\right),{ }^{28} m_{T i} / m_{N e}=2.4$, similar to that for $\mathrm{Zr}$ sputtered in Ar, $m_{Z r} / m_{A r} \sim 2.3$, for which thermalization is also not observed.

With even heavier metal ions, $\mathrm{Hf}$ and $\mathrm{W}$, the original $I_{M e^{+}}\left(E_{i}\right)$ shape with a high-energy tail is maintained even out to 250-300 $\mu$ s, where both $I_{H f^{+}}\left(E_{i}\right)$ and $I_{W^{+}}\left(E_{i}\right)$ exhibit pronounced highenergy tails. For Hf, a low-intensity, low-energy feature at $3-4 \mathrm{eV}$ is also present, indicating that a 
small fraction of emitted ions become thermalized. This is likely related to the fact that $N_{H f^{+}} / N_{A r^{+}} \ll N_{W^{+}} / N_{A r^{+}}$(see Fig. 3).

In general, thermalization is more pronounced for Group IVb than VIb TMs with similar mass (cf. Ti vs. Cr, Zr vs. Mo, and/or Hf vs. W), which indicates that apart from TM atom mass, the sputtering and self-sputtering yields also affect rarefaction. Both $S_{A r \rightarrow M e}$ and $S_{M e \rightarrow M e}$ are higher for VIb metals leading to higher momentum transfer to gas atoms and, hence, stronger rarefaction. These observations are consistent with higher $N_{M e^{+}} / N_{A r^{+}}$values for VIb metals (see Fig. 3), as discussed above.

The results presented here are completely consistent with those obtained from a different sputtering system, utilizing a much smaller $20 \mathrm{~cm}^{2}$ target, with a base pressure of $1.3 \times 10^{-3} \mathrm{mPa}$ $\left(1.0 \times 10^{-8}\right.$ Torr) and an Ar sputtering pressure of 0.4 Pa (3 mTorr). Trends in $N_{M e^{+}} / N_{A r^{+}}$as a function of peak target current density (Fig. 3) have been confirmed for Ti, Zr, Hf, Cr, and Mo targets, which indicates the fundamental nature of the reported phenomena.

\section{CONCLUSIONS}

In summary, we performed energy- and time-dependent mass spectrometry analyses of metal- and gas-ion fluxes incident at the substrate plane during HiPIMS sputtering of Groups-IVb and VIb transition-metal targets in Ar. We showed that the time-and energy-integrated metal/gasion ratio $N_{M e^{+}} / N_{A r^{+}}$increases with increasing peak target current density $J_{T \text {,peak }}$ as a result of gas rarefaction. Moreover, the effect scales with increasing metal-ion mass, resulting in $N_{M e^{+}} / N_{A r^{+}}$ varying by two orders of magnitude from $\sim 1$ for Ti $\left(m_{T i} / m_{A r}=1.20\right)$ to $\sim 100$ for $\mathrm{W}\left(m_{W} / m_{A r}=\right.$ 4.60) with $J_{T, p e a k}$ maintained constant at $1 \mathrm{~A} / \mathrm{cm}^{2}$. Hence, the use of synchronized substrate-bias pulses, in order to reduce the trapped Ar concentration in the growing film, requires precise timing 
for metal/gas combinations with $m_{M e} / m_{g} \sim 1$, while with $m_{M e} / m_{g} \gg 1$, the length of the synchronous bias pulse is controlled by the metal-ion time of flight.

\section{ACKNOWLEDGEMENTS}

Financial support from the Swedish Research Council VR Grant 2014-5790, an Åforsk foundation grant \#16-359, the Swedish Government Strategic Research Area in Materials Science on Functional Materials at Linköping University (Faculty Grant SFO Mat LiU No. 2009 00971), and the Knut and Alice Wallenberg foundation Fellowship Grant and Project funding (KAW 2015.0043) is gratefully acknowledged.

\section{Figure captions}

Fig. 1. (Color Online) Time evolution of the energy-integrated $\mathrm{Ti}^{+}, \mathrm{Ti}^{2+}$, and $\mathrm{Ar}^{+}$ion fluxes $F(t)$ incident at the substrate plane during and after HiPIMS pulses (120 $\mu$ s) while sputtering a Ti target in $\mathrm{Ar}$ at $0.4 \mathrm{~Pa}$ (3 mTorr) as a function of the peak target current density $J_{T, p e a k}$ varied from (a) 0.03 to (b) 0.1 , (c) 0.2 , (d) 0.5 , (e) 1.0 , and (f) $2.0 \mathrm{~A} / \mathrm{cm}^{2}$. Grey curves are target current pulses $J_{T}(t)$ scaled to match the $F_{T i^{+}}(t)$ intensity in order to facilitate comparison.

Fig. 2. (Color Online) Time evolution of the energy-integrated $\mathrm{Me}^{+}, \mathrm{Me}^{2+}$, and $\mathrm{Ar}^{+}$ion fluxes $F(t)$, for which $\mathrm{Me}=\mathrm{Ti}(\mathrm{a}), \mathrm{Zr}$ (b), $\mathrm{Hf}$ (c), $\mathrm{Cr}$ (d), Mo (e), and W (f), incident at the substrate plane during and after HiPIMS pulses $(120 \mu$ s) while sputtering Me targets in Ar at $0.4 \mathrm{~Pa}$ (3 mTorr) with a peak target current density $J_{T, p e a k}=1.0 \mathrm{~A} / \mathrm{cm}^{2}$. Grey curves are target current pulses $J_{T}(t)$ scaled to match the $F_{\mathrm{Me}^{+}}(t)$ intensity in order to facilitate comparison. 
Fig. 3. (Color Online) Time- and energy-integrated metal/gas-ion ratios $N_{M e^{+}} / N_{A r^{+}}$at the substrate plane as a function of peak target current density $J_{T, p e a k}$ during HiPIMS sputtering of Ti, Zr, Hf, Cr, Mo, and W targets in Ar at $0.4 \mathrm{~Pa}$ (3 mTorr).

Fig. 4. (Color Online) $\mathrm{Me}^{+}$ion energy distribution functions (IEDFs) recorded at the substrate position during HiPIMS sputtering of $\mathrm{Me}=\mathrm{Ti}$ (a), Zr (b), Hf (c), $\mathrm{Cr}$ (d), Mo (e), and W (f) targets in Ar at 0.4 Pa (3 mTorr). The IEDFs are acquired during 50- $\mu$ s time intervals over the time period from 0 (pulse ignition) to $300 \mu$ s. The pulse length is $120 \mu$ s. 
Table 1. Relevant parameters for analyzing HiPIMS sputtering of Groups IVb and VIb TM targets in Ar. TM mass $m_{M e}$, atomic radii $r_{g},{ }^{29}$ first and second metal ionization potentials $I P_{M e}^{1}$ and $I P_{M e}^{2}{ }^{22}$ TRIM sputtering and self-sputtering yields $S_{A r \rightarrow M e}$ and $S_{M e \rightarrow M e}$ for incident ion energies (in parantheses) ${ }^{30}$ the number of collisions $\eta$ required to thermalize sputtered TM atoms ejected with $10 \mathrm{eV}$ in the forward direction (see Eq. (1)); the average sputtered TM atom mean-free-path $\lambda ;^{26}$ and the thermalization distance $\lambda_{\text {th }}$ (Eq. (1)) for sputtered TM atoms ejected with $10 \mathrm{eV}$ in the forward direction.

$\left.\begin{array}{|c|c|c|c|c|c|c|c|c|c|}\hline \hline \text { Property } & \begin{array}{c}m_{g} \\ {[\mathrm{amu}]}\end{array} & \begin{array}{c}r_{g} \\ {[\AA]}\end{array}\end{array}\right) \begin{gathered}I P_{M e}^{1}[\mathrm{eV}] \\ \mathrm{TM}\end{gathered}$




\section{References}

${ }^{1}$ V. Kouznetsov, K. Macak, J. M. Schneider, U. Helmersson and I. Petrov, Surf. Coat. Technol. 122, 290 (1999).

${ }^{2}$ K. Sarakinos, J. Alami, S. Konstantinidis, Surf. Coat. Technol. 204, 1661 (2010).

${ }^{3}$ I. Petrov, L. Hultman, J.-E. Sundgren, and J. E. Greene, J. Vac. Sci. Technol. A 10, 265 (1992).

${ }^{4}$ I. Petrov, P.B. Barna, L. Hultman, J.E. Greene, J. Vac. Sci. Technol. A 21, 117 (2003).

${ }^{5}$ K. Macak, V. Kouznetsov, J. Schneider, U. Helmersson, I. Petrov, J. Vac. Sci. Technol. A 18, 1533 (2000).

${ }^{6}$ G. Greczynski, J. Lu, J. Jensen, I. Petrov, J.E. Greene, S. Bolz, W. Kölker, Ch. Schiffers, O. Lemmer and L. Hultman, J. Vac. Sci. Technol. A 30, 061504 (2012).

${ }^{7}$ G. Greczynski, J. Lu, J. Jensen, S. Bolz, W. Kölker, Ch. Schiffers, O. Lemmer, J.E. Greene, and L. Hultman, Surf. Coat. Technol. 257, 15 (2014).

${ }^{8}$ G. Greczynski, J. Lu, O. Tengstrand, I. Petrov, J.E. Greene, and L. Hultman, Scripta Mat. 122, 40 (2016).

${ }^{9}$ G. Greczynski, J. Lu, M. Johansson, J. Jensen, I. Petrov, J.E. Greene, and L. Hultman, Surf. Coat. Technol. 206, 4202 (2012).

${ }^{10}$ G. Greczynski, J. Lu, M. Johansson, J. Jensen, I. Petrov, J.E. Greene, and L. Hultman, Vacuum 86, 1036 (2012).

${ }^{11}$ G. Greczynski, J. Lu, J. Jensen, I. Petrov, J.E. Greene, S. Bolz, W. Kölker, Ch. Schiffers, O. Lemmer and L. Hultman, Thin Solid Films 556, 87 (2014).

${ }^{12}$ G. Greczynski, J. Patscheider, J. Lu, B. Alling, A. Ektarawong, J. Jensen, I. Petrov, J. E. Greene, L. Hultman, Surf. Coat. Technol. 280, 174 (2015).

${ }^{13}$ G. Greczynski, S. Mráz, M. Hans, D. Primetzhofer, J. Lu, L- Hultman, J.M. Schneider, J. Appl. Phys. 121, 171907 (2017).

${ }^{14}$ G. Greczynski, J. Lu, S. Bolz, W. Kölker, Ch. Schiffers, O. Lemmer, I. Petrov, J.E. Greene, L. Hultman, J. Vac. Sci. Technol. A 32, 041515 (2014).

${ }^{15}$ H. Fager, O. Tengstrand, J. Lu, S. Bolz, B. Mesic, W. Kölker, Ch. Schiffers, O. Lemmer, J. E. Greene, L. Hultman, I. Petrov, G. Greczynski, J. Appl. Phys. 121, 171902 (2017).

${ }^{16}$ G. Greczynski and L. Hultman, Vacuum 84, 1159 (2010).

${ }^{17}$ J. Bohlmark, M. Lattemann, J.T. Gudmundsson, A.P. Ehiasarian, Y.A. Gonzalvo, N. Brenning, U. Helmersson, Thin Solid Films 515, 1522 (2006).

${ }^{18}$ S. Kadlec, Plasma Proc. Polymers 4, S419 (2007).

${ }^{19}$ C. Vitelaru, D. Lundin, G. D. Stancu, N. Brenning, J. Bretagne, and T. Minea, Plasma Sources Science and Technology 21, 025010 (2012).

${ }^{20}$ A. Kanitz, A. Hecimovic, M. Böke, and J. Winter, Journal of Physics D: Applied Physics 49, 125203 (2016).

${ }^{21}$ D. Horwat, A. Anders, J. Appl. Phys. 108, 123306 (2010).

${ }^{22}$ David R. Lide (ed), CRC Handbook of Chemistry and Physics, 84th Edition. CRC Press. Boca Raton, Florida, 2003; Section 10, Atomic, Molecular, and Optical Physics; Ionization Potentials of Atoms and Atomic Ions.

${ }^{23}$ P. Sigmund, J. Vac. Sci. Technol. A 17, 396 (1979).

${ }^{24}$ M. W. Thompson, Physics Reports 69, 335 (1981).

${ }^{25}$ J.T. Gudmundsson, N. Brenning, D. Lundin, and U. Helmersson, J. Vac. Sci. Technol. A 30, 030801 (2012).

${ }^{26}$ W.D. Westwood, J. Vac. Sci. Technol. 15, 1 (1978).

${ }^{27}$ J.H. Jeans, ”The Dynamical Theory of Gases”, Dover New York, 1954

${ }^{28}$ G. Greczynski, I. Petrov, J.E. Greene, and L. Hultman, Vacuum 116, 36 (2015).

${ }^{29}$ J.C. Slater, J. Chem. Phys. 41, 3199 (1964).

${ }^{30}$ J. F. Ziegler, J. P, Biersack, U. Littmark, "The Stopping and Range of Ions in Solids", Pergamon Press, New York (1984) 


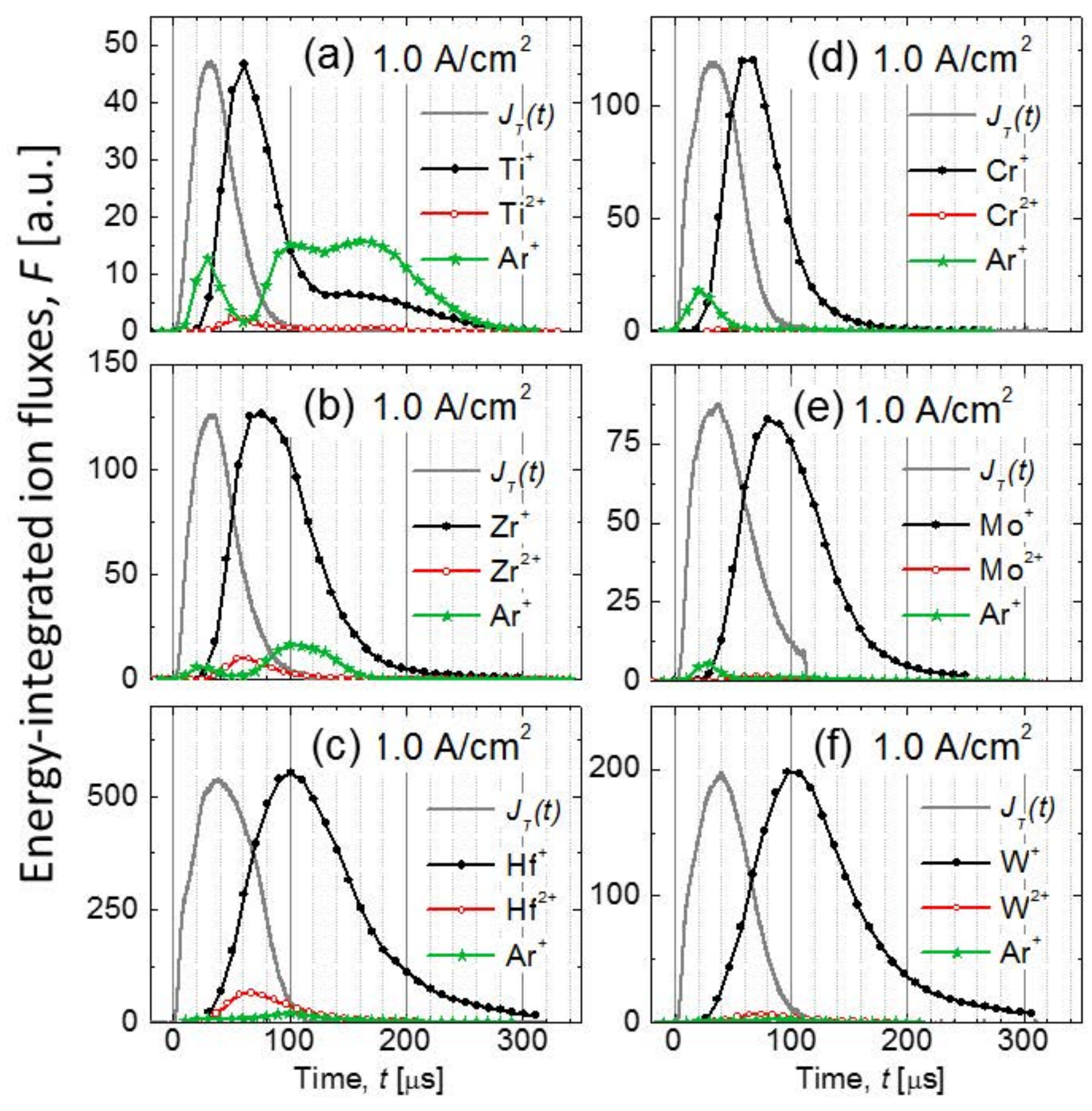




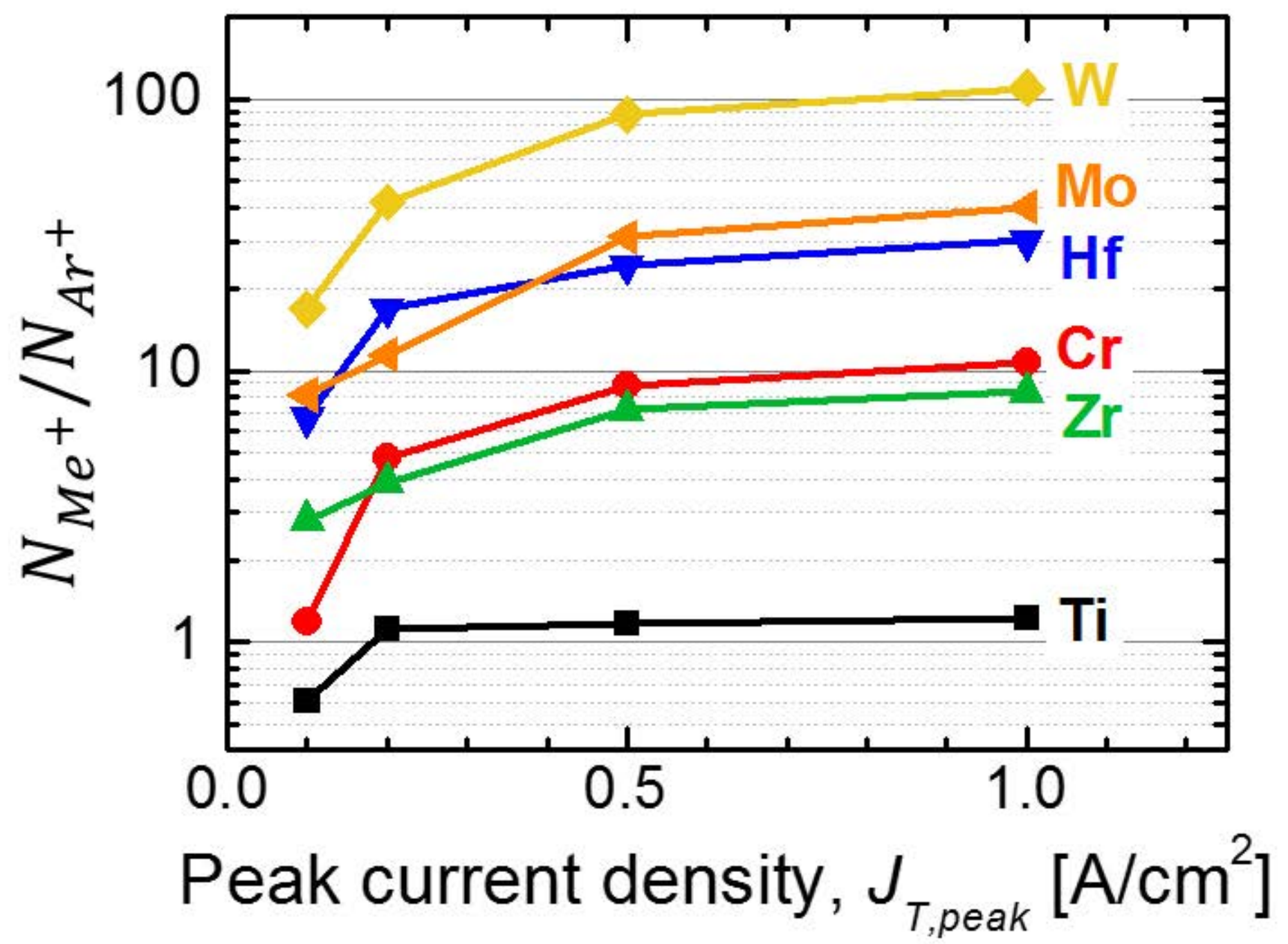




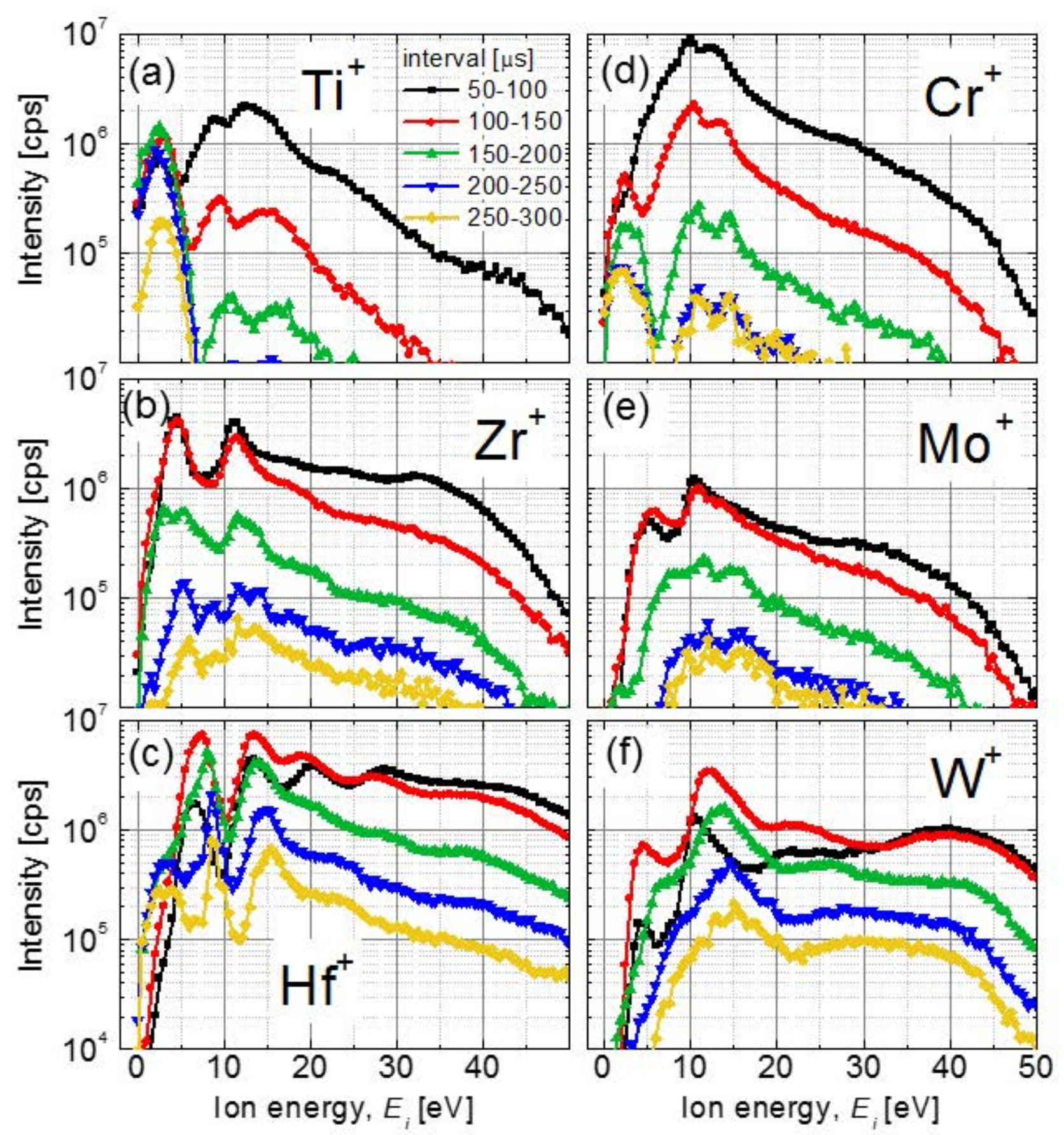




\begin{tabular}{|c|c|c|c|c|c|c|c|c|c|c|}
\hline TM & Property & $\begin{array}{c}m_{g} \\
{[\mathrm{amu}]}\end{array}$ & $\begin{array}{c}r_{g} \\
\text { [Å] }\end{array}$ & $\begin{array}{c}I P_{M e}^{1} \\
{[\mathrm{eV}]}\end{array}$ & $\begin{array}{c}I P_{M e}^{2} \\
{[\mathrm{eV}}\end{array}$ & $S_{A r \rightarrow M e}$ & $S_{M e \rightarrow M e}$ & 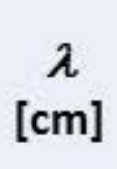 & $\begin{array}{c}\eta \\
(10 \mathrm{eV})\end{array}$ & $\begin{array}{c}\lambda_{t h} \\
(10 \mathrm{eV}) \\
{[\mathrm{cm}]}\end{array}$ \\
\hline & $\mathrm{Ti}$ & 47.87 & 1.40 & 6.85 & 13.62 & $\begin{array}{c}0.62 \\
(480)\end{array}$ & 0.51 & 2.5 & 3.7 & 9.3 \\
\hline & $\mathrm{Zr}$ & 91.22 & 1.55 & 6.66 & 13.21 & $\begin{array}{c}0.77 \\
(440)\end{array}$ & 0.51 & 1.8 & 6.2 & 11.2 \\
\hline & Hf & 178.49 & 1.55 & 6.85 & 14.98 & $\begin{array}{c}1.18 \\
(580)\end{array}$ & 0.86 & 1.4 & 11.3 & 15.8 \\
\hline & $\mathrm{Cr}$ & 52.00 & 1.4 & 6.79 & 16.54 & $\begin{array}{c}1.59 \\
(600)\end{array}$ & 1.34 & 2.4 & 4.0 & 9.6 \\
\hline & Mo & 95.94 & 1.45 & 7.09 & 16.16 & $\begin{array}{l}1.12 \\
(520)\end{array}$ & 0.72 & 1.9 & 6.4 & 12.2 \\
\hline & w & 183.84 & 1.35 & 8.01 & 17.68 & $\begin{array}{c}1.25 \\
(590)\end{array}$ & 0.91 & 1.6 & 11.3 & 18.1 \\
\hline
\end{tabular}

